\title{
Local lateral environment of the molecules at the surface of DMSO-water mixtures
}

Balázs Fábián, ${ }^{1,2}$ Abdenacer Idrissi, ${ }^{3}$ Bogdan Marekha, ${ }^{3}$ and Pál Jedlovszky ${ }^{4,5,6^{*}}$

${ }^{1}$ Department of Inorganic and Analytical Chemistry, Budapest University of Technology and Economics, Szt. Gellért tér 4, H-1111 Budapest, Hungary

${ }^{2}$ Institut UTINAM (CNRS UMR 6213), Université de Franche-Comté, 16 route de Gray, F-25030 Besançon, France

${ }^{3}$ Laboratoire de Spectrochimie Infrarouge et Raman (UMR CNRS A8516), Université Lille 1, Science et Technologies, 59655 Villeneuve d'Ascq Cedex, France

${ }^{4}$ EKF Department of Chemistry, Leányka utca 6, H-3300 Eger, Hungary

${ }^{5}$ MTA-BME Research Group of Technical Analytical Chemistry, Szt. Gellért tér 4, H-1111 Budapest, Hungary

${ }^{6}$ Laboratory of Interfaces and Nanosize Systems, Institute of Chemistry, Eötvös Loránd University, Pázmány P. Stny 1/A, H-1117 Budapest, Hungary

*E-mail: pali@ektf.hu 
This document is the Accepted Manuscript version of a Published Work that appeared in final form in J. Phys.: Condens. Matter 28 (2016) 404002 (10pp), copyright @ IOP Science after peer review and technical editing by the publisher. To access the final edited and published work see http://iopscience.iop.org/article/10.1088/0953$\underline{8984 / 28 / 40 / 404002}$

\section{Abstract:}

Molecular dynamics simulations of the liquid-vapour interface of dimethyl sulphoxide (DMSO)water mixtures of 11 different compositions, including the two neat systems are performed on the canonical $(N, V, T)$ ensemble at $298 \mathrm{~K}$. The molecules constituting the surface layer of these systems are selected by means of the identification of the truly interfacial molecules (ITIM) method, and their local lateral environment at the liquid surface is investigated by performing Voronoi analysis. The obtained results reveal that both molecules prefer to be in mixed local environment, consisting of both kinds of molecules, at the liquid surface, and this preference is even stronger here than in the bulk liquid phase. Neat-like patches, in which a molecule is surrounded by like neighbours, are not found, however, vacancies that are surrounded solely by water molecules are observed at the liquid surface. Our results show that strongly hydrogen bonded DMSO $\cdot \mathrm{H}_{2} \mathrm{O}$ complexes, known to exist in the bulk phase of these mixtures, are absent from the liquid surface. 
This document is the Accepted Manuscript version of a Published Work that appeared in final form in J. Phys.: Condens. Matter 28 (2016) 404002 (10pp), copyright @ IOP Science after peer review and technical editing by the publisher. To access the final edited and published work see http://iopscience.iop.org/article/10.1088/0953$\underline{8984 / 28 / 40 / 404002}$

\section{Introduction.}

Mixtures of dimethyl sulphoxide (DMSO) and water are widely used in a number of fields of chemistry and chemical industry [!1], e.g., as the medium of various reactions in organic synthesis [!2-5] and electrochemistry [!6], as cryoprotectors [!7], in separation science [!8], or in biotechnology [!9] and cell biology [!10]. The broad range of applications of these mixtures is strongly related to the fact that a number of their physical-chemical properties, such as viscosity $[! 11,12]$, static dielectric permittivity $[! 13,14]$, refractive index $[! 15,16]$ or various thermodynamic data [!17-22] exhibit non-linear composition dependence, and often even anomalous behaviour. Thus, for instance, these mixtures show extremely large freezing point depression: the freezing point of the mixture containing 25 mole \% DMSO is 88 and $70 \mathrm{~K}$ below that of neat DMSO and neat water, respectively [!23,24]. Similarly, the dielectric constant [!13] as well as dielectric relaxation times [!25] go through a maximum as a function of the composition.

To understand the origin of these anomalies, the structure of DMSO-water mixtures has been extensively studied both by experimental methods [!11-40] and computer simulations [!41-54] in the past decades. These studies revealed that DMSO does not alter the local structure of the hydrogen bonding network in water, in other words, it is neither a structure making nor a structure breaking solute [!28,32]. It is also clear that the full miscibility of the two components is primarily caused by the energy decrease upon mixing [!17,18,52]. Entropy, on the other hand, increases when neat DMSO is diluted by water, but decreases when neat water is diluted by DMSO [!18,52]. As a consequence, in a clear contrast with mixtures whose miscibility is solely of entropic origin, such as those of methanol and acetone [!55], DMSO-water mixtures do not show microheterogeneous structure, instead, both molecules prefer to be surrounded by unlike rather than like neighbours [!53]. The unusual strength of the DMSO-water hydrogen bonds results in strongly hydrogen bound DMSO:water complexes of various stoichiometry, such as 1:2 [!20,35,42,45,46,48], 2:3 [!46] and $1: 3$ [!54] although the existence of the latter complex is still under debate [!46]. The question whether such complexes involve all the water or DMSO molecules of the system [!14], they are in an equilibrium with "monomeric" molecules [!45,46,53], or the molecular structure of these systems can be described in terms of a homogeneous hydrogen bonded network rather than as a mixture of well-defined molecular complexes [!30] has not been settled either. The reason why these questions are still open is related to the fact that DMSO:water complexes were scarcely observed directly; their existence was usually hypothesised in explaining anomalous properties of 
This document is the Accepted Manuscript version of a Published Work that appeared in final form in J. Phys.: Condens. Matter 28 (2016) 404002 (10pp), copyright @ IOP Science after peer review and technical editing by the publisher. To access the final edited and published work see http://iopscience.iop.org/article/10.1088/0953$\underline{8984 / 28 / 40 / 404002}$

these mixtures, or assumed on the basis of indirect evidences, such as certain features of various pair correlation functions $[! 14,42,43,45]$.

Surprisingly, in a clear contrast with the wealth of studies concerning the bulk liquid phase of DMSO-water mixtures, little is known about the molecular structure of their surface, in spite of the fact that unusual bulk phase properties might well lead to unusual surface properties of these mixtures. Experimental investigations include second harmonic generation (SHG) and vibrational sum frequency generation (SFG) spectroscopic studies [!56-59], which led to the general conclusions that DMSO is adsorbed at the surface of these mixtures, [!56,57] and the presence of DMSO does not influence the hydrogen bonding structure of the water molecules even at the liquid surface [!58]. Using a mean-field statistical mechanical model Luzar investigated the extent of hydrogen bonding of the molecules as a function of the distance from the liquid surface, and explained the excess surface tension by the contribution of the hydrogen bonding interaction of the unlike molecule pairs [!60]. Computer simulation studies of DMSO-water mixtures also found that DMSO exhibits stronger affinity to the liquid surface than water $[! 59,61,62]$. Benjamin calculated the potential of mean force that governs DMSO adsorption at the surface of its infinitely dilute aqueous solution [!61], whereas Pojják et al. showed that this adsorption affects solely the first molecular layer at the liquid surface [!62]. We are not aware of, however, any study concerning the local lateral environment of the water and DMSO molecules at the surface of their mixtures.

In analysing surface properties of a liquid phase in computer simulation, i.e., when the system is treated in atomistic resolution one has to face to the difficulty that the liquid surface is corrugated, on the molecular length scale, by capillary waves. This effect has to be removed before any analysis is performed. In other words, one has to find first the set of the truly interfacial molecules, i.e., the ones that are located right at the boundary of the two phases. Neglecting the effect of the capillary waves leads to the analysis of a subset of molecules that is different from that of the surface ones, which introduces a systematic error of unknown magnitude in any surfacerelated result [!62-64]. This error might even propagate to the thermodynamic properties of the system studied [!65]. To overcome this difficulty several methods have been proposed in the past one and a half decades [!63,66-72], among which the identification of the truly interfacial molecules (ITIM) [!63] turned out to be an excellent compromise between computational cost and accuracy [!71].

In this paper we present a detailed analysis of the local lateral structure around the molecules at the surface of DMSO-water mixtures of different compositions on the basis of molecular 
This document is the Accepted Manuscript version of a Published Work that appeared in final form in J. Phys.: Condens. Matter 28 (2016) 404002 (10pp), copyright @ IOP Science after peer review and technical editing by the publisher. To access the final edited and published work see http://iopscience.iop.org/article/10.1088/0953$\underline{8984 / 28 / 40 / 404002}$

dynamics computer simulation. For this purpose, we perform a two-dimensional Voronoi analysis $[! 73,74]$ considering the surface molecules, which are identified in terms of the ITIM method. The method of the Voronoi polygons (or, in three dimensions, that of the Voronoi polyhedra) [!75] is a very powerful tool in characterising the local molecular environment in computer simulations. Since in a two dimensional system of discrete seeds (e.g., molecules) the Voronoi polygon (VP) of a seed is the locus of the planar points that are closer to this seed than to any other one, the area of a VP represents the portion of the plane that is occupied by its central seed (molecule). The shape of a VP is characteristic of the neighbourhood of its central seed, whereas VP vertices, being the planar points at which the distance from the nearest seed is of local maximum, are the centres of the largest circular voids located between the seeds. Recently we performed such an analysis of the local environment of the molecules in the bulk phase of DMSO-water mixtures [!53]. The present study extends this investigation to the surface of these systems.

The paper is organised as follows. In section 2 details of the calculations performed, including molecular dynamics simulations, ITIM analysis and Voronoi analysis are given. The obtained results are discussed in detail in section 3. Finally, in section 4 the main conclusions of this study are summarised.

\section{Computational details}

\subsection{Molecular dynamics simulations}

We employ molecular dynamics (MD) simulations to study the local lateral structure around the molecules at the liquid-vapour interface of DMSO-water mixtures at 11 different compositions, including the two neat systems. The simulations were carried out on the canonical $(N, V, T)$ ensemble at $298 \mathrm{~K}$. A rectangular basic MD cell with $X, Y$ and $Z$ edges of 300, 50 and $50 \AA$, respectively, $(X$ being the macroscopic surface normal axis) was used. All the studied systems contained 4000 molecules in total, among which 0, 120, 200, 400, 800, 1200, 1600, 2000, 2400, 3400, and 4000 were DMSO. We will use the corresponding bulk mole fraction notation and these systems will be referred to in this paper as the DMSO000, DMSO003, DMSO005, DMSO010, DMSO020, DMSO030, DMSO040, DMSO050, DMSO060, DMSO085, and DMSO100 system, respectively.

In one of our previous works [!52] it was shown that the combination of the DMSO forcefield model developed by Vishnyakov, Lyubartsev and Laaksonen (VLL) [!46] and the TIP4P water 
This document is the Accepted Manuscript version of a Published Work that appeared in final form in J. Phys.: Condens. Matter 28 (2016) 404002 (10pp), copyright @ IOP Science after peer review and technical editing by the publisher. To access the final edited and published work see http://iopscience.iop.org/article/10.1088/0953$\underline{8984 / 28 / 40 / 404002}$

model [!76] gives the best reproduction of the thermodynamic mixing properties over the whole concentration range. In this regard we employ this combination of models in the present work as well. Both molecules were treated as rigid; their geometry was maintained by means of the LINCS algorithm [!77]. The VLL model of DMSO represents the methyl groups as united atoms [!46], whereas the TIP4P model of water has a separate non-atomic interaction site carrying the negative fractional charge [!76]. The interaction energy between the molecules was represented as a pairwise sum of the Lennard-Jones and Coulomb interactions of all pairs of their interaction sites. The main interaction parameters, such as $\varepsilon$ and $\sigma$ values of the Lennard-Jones potential as well as fractional charges $(q)$ of all the interaction sites are given in Table 1. Conventional LorentzBerthelot combination rules [!78] were used to calculate the Lennard-Jones interaction energy between a pair of unlike interaction sites. All the interactions were truncated to zero if two molecules were more than $15 \AA$ apart (the $\mathrm{O}$ and $\mathrm{S}$ atoms being regarded as the representative centres of the water and DMSO molecules, respectively). Smooth Particle Mesh Ewald (SPME) method [!79] was used to calculate the long-range parte of electrostatic interactions.

All MD simulations were performed using the GROMACS 4.5.5 program suite [!80]. Newtonian equations-of-motions were integrated in time steps of $1 \mathrm{fs}$. Nosé-Hoover thermostat $[! 81,82]$ with a time constant of 1 ps was used to keep temperature constant. In preparing initial configuration the required number of molecules were first randomly placed in a basic box whose $Y$ and $Z$ edges were already set $50 \AA$ long, while the length of the $X$ axis roughly corresponded to the bulk liquid phase density of the system simulated. After preliminary energy minimisations the systems were anisotropically equilibrated for $2 \mathrm{~ns}$ on the isothermal-isobaric $(N, p, T)$ ensemble at 1 bar in such a way that only the $X$ edge of the basic box has been allowed to change. Pressure was controlled by means of the Parrinello-Rahman barostat [!83], using a time constant of 1 ps. The interface was created by increasing the $X$ edge length to its final value of $300 \AA$. The interfacial systems were additionally further equilibrated, now on the canonical ensemble, for at least $4.5 \mathrm{~ns}$. Conventional three-dimensional periodic boundary conditions were employed both in the bulk phase and in the interfacial simulations. Finally, 2000 equilibrium configurations per system were produced for subsequent analysis from $2 \mathrm{~ns}$ long production runs with the sampling time of $1 \mathrm{ps}$. 
This document is the Accepted Manuscript version of a Published Work that appeared in final form in J. Phys.: Condens. Matter 28 (2016) 404002 (10pp), copyright @ IOP Science after peer review and technical editing by the publisher. To access the final edited and published work see http://iopscience.iop.org/article/10.1088/0953$\underline{8984 / 28 / 40 / 404002}$

\subsection{Determination of the surface layer by ITIM analysis}

The detailed description of the ITIM methodology is available in the original publication, [!63] therefore here we will only briefly summarize the basics. In order to identify all the truly interfacial molecules of a given phase within the framework of ITIM analysis a spherical probe particle of a given size approaches the phase of interest along a virtual test line, which is normal to the macroscopic interface, from the opposite phase, i.e., from the vapour to the liquid phase in our case [!63]. The first molecule of the phase of interest 'touched' by the approaching probe particle is considered as being interfacial. The probe then starts moving along the next test line. The final set of truly interfacial molecules is completed when all the test lines are considered.

In this study a probe sphere of the radius of $R_{\mathrm{p}}=2 \AA$ has been used, following the notion that the probe size should be comparable with that of the particles constituting the system studied. [!63] In defining touching positions with the probe the atoms have been represented by spheres whose diameters corresponded to their Lennard-Jones distance parameters (see Table 1). Once any atom of a molecule has been touched by the probe the entire molecule has been marked as being at the interface. Test lines have been arranged in a $100 \times 100$ grid in the macroscopic plane of the interface, $Y Z$, thus, the distance of two neighbouring grids have been $0.5 \AA$. This choice of the grid spacing is justified by our earlier finding that it already leads to convergent results. [!71] Finally, all results have been averaged over the two liquid-vapour interfaces present in the basic simulation box. An equilibrium snapshot of the surface of the DMSO020 system is shown in Figure 1 as obtained from the simulation.

\subsection{Voronoi analysis}

To perform the two dimensional Voronoi analysis of the surface molecules, first the positions of their central atoms (i.e., O for water and S for DMSO) have been projected to the macroscopic plane of the interface, $Y Z$. Obviously, the two liquid-vapour interfaces present in the basic box have been treated separately; hence, the 2000 sample configurations collected per system provided a set of 4000 different projections. Two-dimensional Voronoi analysis has then been performed on the set of these planar projections.

In performing the Voronoi analyses a two-dimensional variant of the algorithm proposed by Ruocco, Sampoli, and Vallauri [!84] has been used. When the VP around a given seed (i.e., projection of the centre of an interfacial molecule to $Y Z$ ) was determined, we did not use any information obtained when constructing the VP of another seed. This treatment provides an easy 
This document is the Accepted Manuscript version of a Published Work that appeared in final form in J. Phys.: Condens. Matter 28 (2016) 404002 (10pp), copyright @ IOP Science after peer review and technical editing by the publisher. To access the final edited and published work see http://iopscience.iop.org/article/10.1088/0953$\underline{8984 / 28 / 40 / 404002}$

way of checking the consistency of the calculation. Namely, the sum of the area of the individual VP in a sample configuration has to be equal with the $Y Z$ cross section of the basic box of $2500 \AA^{2}$. The two values indeed agreed with each other within the numerical precision of the calculation in every case. In determining the VP around a given seed first we considered a tentative polygon that is large enough to contain the real, yet unknown, VP entirely. For simplicity, we have used the entire $Y Z$ cross section of the basic box, centred on the given seed, as this tentative polygon. Then the other seeds in the system have been sorted according to their distance from the central one, and the tentative polygon has been gradually refined in the following procedure, starting with the nearest seed and proceeding with progressively farther ones. For each of these seeds it is checked whether the orthogonal bisector between this seed and the central one cuts the tentative polygon to two parts or not. In the first case, the new tentative polygon is that part of the original one which still contains the central seed, whereas in the second case the tentative polygon is left unaltered. The procedure stops when the next seed to be considered is at least twice as far from the central seed than the farthest vertex of the polygon, since from this point on the orthogonal bisectors cannot cut the tentative polygon any more, and hence this tentative polygon is the real VP of the central seed [!84].

Once a VP is determined, its vertices have been sorted according to their sequence along the perimeter, and the VP has been divided to $\left(N_{\mathrm{v}}-2\right)$ elemental triangles, defined by its first, $i$ th and $(i+1)$ th vertex, where $N_{\mathrm{v}}$ is the number of vertices pertaining to this VP. The area of the VP, $A$, has then been calculated as the sum of the area of these elemental triangles:

$$
A=\frac{1}{2} \sum_{i=1}^{N_{v}-2}\left|\left(\mathbf{r}_{\mathbf{i}}-\mathbf{r}_{\mathbf{1}}\right) \times\left(\mathbf{r}_{\mathbf{i}+\mathbf{1}}-\mathbf{r}_{\mathbf{1}}\right)\right|
$$

where $\mathbf{r}_{1}, \mathbf{r}_{\mathbf{i}}$, and $\mathbf{r}_{\mathbf{i}+\mathbf{1}}$ are the position vectors of the first, $i$ th and $(i+1)$ th vertex, respectively, in a coordinate frame the origin of which is the central seed. The shape of a VP is characterised by the acircularity parameter, $\phi[! 85]$, defined in analogy with the three-dimensional asphericity parameter $[! 84]$ as

$$
\phi=\frac{1}{4 \pi} \frac{L^{2}}{A}
$$

$L$ being the circumference length of the VP. The value of $\phi$ is unity for a perfect circle, and the less spherical the VP is, the larger is its value. Thus, for instance, the acircularity parameter values 
This document is the Accepted Manuscript version of a Published Work that appeared in final form in J. Phys.: Condens. Matter 28 (2016) 404002 (10pp), copyright @ IOP Science after peer review and technical editing by the publisher. To access the final edited and published work see http://iopscience.iop.org/article/10.1088/0953$\underline{8984 / 28 / 40 / 404002}$

corresponding to a perfect hexagon, square, and equilateral triangle are 1.103, 1.273, and 1.654, respectively. Finally, the radii of the (circular) vacancies located between the seeds, $R$, can simply be obtained as the distance of the VP vertices from the corresponding central seeds.

\section{Results and discussion}

Before performing Voronoi analyses, we have determined the composition of the surface layer of the different DMSO-water mixtures. The composition of the surface layer (in terms of DMSO mole fraction) is shown as a function of the bulk phase composition in Figure 2, whereas the bulk phase and surface DMSO mole fraction values obtained in the different systems simulated are collected in Table 2. As is seen, DMSO is indeed adsorbed considerably strongly at the liquid surface, and this adsorption is stronger in mixtures of lower DMSO content; the DMSO mole fraction at the surface of its dilute solutions can be even an order of magnitude higher than that in the bulk liquid phase. This result is agreement with those obtained earlier by Allen et al. using SFG spectroscopy [57]. Since in this study we are interested in the local lateral environment of the molecules within the surface layer, in the following, we discuss the results obtained from the Voronoi analysis in terms of the surface layer composition rather than that of the bulk liquid phase or the entire system simulated. To check how sensitively our results depend on the particular choice of the probe sphere radius, $R_{\mathrm{p}}$, used in the ITIM analysis, we have repeated this calculation with the $R_{\mathrm{p}}$ values of $1.0 \AA$, $1.5 \AA, 2.5 \AA$ and $3.0 \AA$ besides the $R_{\mathrm{p}}$ value of $2.0 \AA$ used in the entire analysis. The results, shown in the inset of Fig. 2 clearly confirm the weak dependence of the results on the actual value of $R_{\mathrm{p}}$ given that it falls in the size range of the particles. In the following, only the results obtained with the $R_{\mathrm{p}}$ value of $2.0 \AA$ are shown.

The area distribution of the VP of the surface molecules, $P(A)$, is shown in Figure 3 as obtained in all systems simulated. Although the obtained distributions always show one single peak, in some cases they can only be fitted well by the sum of two Gaussian functions, whereas fitting these data by one single Gaussian leaves some of their features unreproduced. This is illustrated in Figure 4, showing the fit of the $P(A)$ data, obtained in the DMSO005 system (corresponding to the DMSO mole fraction of about 0.28 in the surface layer, see Table 2), by one single Gaussian as well as by the sum of two Gaussian functions. The peak positions of the two individual Gaussian functions as well as their relative weights in the fitting function are shown in Figure 5. As is seen, 
This document is the Accepted Manuscript version of a Published Work that appeared in final form in J. Phys.: Condens. Matter 28 (2016) 404002 (10pp), copyright @ IOP Science after peer review and technical editing by the publisher. To access the final edited and published work see http://iopscience.iop.org/article/10.1088/0953$\underline{8984 / 28 / 40 / 404002}$

the position of both peaks increases steadily with increasing DMSO concentration, reflecting the well visible shift of the $P(A)$ distribution peak. Further, the relative weight of the first peak decreases, while that of the second peak increases with increasing DMSO concentration up to the DMSO surface mole fraction of about 0.6, above which the first peak vanishes completely and the obtained distribution becomes of Gaussian shape.

The obtained picture is considerably different from both what was previously seen in the bulk liquid phase of DMSO-water mixtures [!53] and what was obtained in systems of microheterogeneous structure, such as water-urea [!86] and acetone-methanol [!87] mixtures. Thus, in the latter systems the position of the two peaks does not depend on the composition, reflecting the fact that the majority of the molecules are surrounded by like neighbours, and hence experience neat-like local environment. Although the two components of such mixtures are evidently miscible, the thermodynamic driving force behind their miscibility is the entropy gain rather than the lowering of the energy [!55], which results in the fact that molecules prefer to be in contact with like neighbours. The mixing of DMSO and water, on the other hand, is driven by the energy decrease [!52], resulting in the preference of the molecules for being in mixed local environments [!53]. In the bulk liquid phase of DMSO-water mixtures, however, the VP volume distribution exhibits four distinct peaks, the position of two of which turned out to be composition independent, while that of the other two is shifting gradually to higher values with increasing DMSO concentration [!53]. The peaks of the first kind correspond to molecules that are in a local environment formed by like neighbours, while the peaks that are composition dependent reflect the contribution of the molecules that are in a mixed local environment. [!53] As is seen from Fig. 5, at the liquid surface only these latter peaks are present, indicating that both molecules prefer to be in a mixed lateral environment even at the liquid surface, moreover, this preference is even stronger than in the bulk liquid phase. The peak occurring at lower $A$ values reflects the contribution of the water, while that at higher areas the contribution of the DMSO molecules, and their gradual shift to higher area values with increasing DMSO concentration reflects the increasing fraction of DMSO neighbours in these mixed local lateral environments.

Similar conclusions can be drawn from the analysis of the acircularity parameter distribution, $P(\phi)$, of the VP of the surface molecules (see Figure 6). Thus, the distribution of the acircularity parameter can always be reasonably well fitted by one single Gaussian function, and the fit cannot be substantially improved by introducing a second Gaussian in the fitting procedure. The slight asymmetry of the obtained $P(\phi)$ distributions, reflected in the somewhat elongated tail at the 
This document is the Accepted Manuscript version of a Published Work that appeared in final form in J. Phys.: Condens. Matter 28 (2016) 404002 (10pp), copyright @ IOP Science after peer review and technical editing by the publisher. To access the final edited and published work see http://iopscience.iop.org/article/10.1088/0953$\underline{8984 / 28 / 40 / 404002}$

large $\phi$ side of the peak is thus not a sign of the presence of two markedly different local lateral environments, instead, it is probably the simple consequence of the fact that the surface layer itself is not completely planar, and hence the local environment of the molecules located at surface portions of different curvatures are slightly differently distorted when projecting them to the macroscopic plane of the interface, $Y Z$. Further, the position of the $P(\phi)$ peak does not show considerable composition dependence, it is located at the $\phi$ value of 1.24 at the surface of neat water and at $\phi=1.21$ at the surface of neat DMSO. When comparing this result to the asphericity parameter distributions observed previously in the bulk liquid phase of DMSO-water mixtures [!53], similar conclusions can be drawn then when the $P(A)$ distributions obtained at the liquid surface and VP volume distributions obtained in the bulk liquid phase are compared. Namely, in bulk DMSO-water mixtures the asphericity parameter distribution resulted in three distinct peaks, two of which being characteristic to the two neat-like local environments, while the third one to the mixed local environment [!53]. At the liquid surface, on the other hand, only the peak characteristic to the mixed local lateral environment can be observed. Finally, it should be noted that this mixed local lateral environment results in two markedly different peaks of the $P(A)$ distribution, but only in one single peak of the acircularity parameter distribution. The reason of this difference is simply that the VP area evidently depends also on the size of its central particle (and, hence, different characteristic values correspond to the water and DMSO molecules that are in the same mixed local lateral environment), while the acircularity parameter of a VP is size independent, and reflects solely the properties of the local environment, irrespectively of the type of its central particle.

The picture emerging from these analyses can be further refined by analysing the distribution of the radius of the largest circular vacancies located between the molecules at the liquid surface, $P(R)$. The obtained distributions, shown in Figure 7, can always be very well fitted by one single Gaussian function, as it is illustrated in the inset of Fig. 7 on the example of the DMSO020 system. The position of the peak of the $P(R)$ distribution shifts to larger values with increasing DMSO concentration (shown by the arrow in Fig. 7), reflecting simply the fact that larger voids can exist between the larger DMSO molecules than between water. The Gaussian shape of the $P(R)$ distributions corresponding to every compositions indicates again that both molecules are rather uniformly distributed at the liquid surface, no large self-associates exist among them. (In the presence of such self-associates, the $P(R)$ distribution should also have peaks that are characteristic of the neat-like local environments, located at the $R$ values of the $P(R)$ distribution peaks of the corresponding neat liquids.) On the other hand, the gradual shift of the peak position 
This document is the Accepted Manuscript version of a Published Work that appeared in final form in J. Phys.: Condens. Matter 28 (2016) 404002 (10pp), copyright @ IOP Science after peer review and technical editing by the publisher. To access the final edited and published work see http://iopscience.iop.org/article/10.1088/0953$\underline{8984 / 28 / 40 / 404002}$

from the value characteristic of neat water to that of neat DMSO reflects the increasing fraction of DMSO molecules around these voids.

All the analyses performed so far have led to results that are consistent with the mixed local environment of the molecules. No sign of the existence of neat-like patches at the surface of DMSO-water mixtures has been evidenced so far. This finding shows a clear contrast with what was seen in the bulk liquid phase of these mixtures, where, besides the dominance of the mixed local environment, traces of neat-like water regions were also observed [!53]. To find out whether such patches do exist, although in a small extent, even at the liquid surface, or they are indeed completely absent here, a very sensitive analysis is the calculation of the $P(R)$ distributions by systematically disregarding one of the two components in the analysis [!53]. Thus, when one of the two components is disregarded, the area they occupy is transformed to voids, and will contribute to the $P(R)$ distribution.

The $P(R)$ distributions obtained by taking only the DMSO molecules into account and disregarding waters, and by taking only the water molecules into account and disregarding DMSO are shown in Figures 8 and 9, respectively. The two sets of $P(R)$ distributions show markedly different behaviour, being rather similar to the results obtained in the bulk phase of DMSO-water mixtures [!53]. Thus, when only DMSO molecules are taken into account, the obtained $P(R)$ curves are always of Gaussian shape, getting broader and their position being shifted to larger $R$ values with decreasing DMSO concentration (as indicated by the arrow in Fig. 8). These changes reflect the trivial fact that DMSO is gradually diluted: if less DMSO molecules are at the liquid surface the voids between them are obviously larger.

A somewhat different picture is seen; however, when only water molecules are taken into account in the analysis, and DMSO is disregarded. In this case, some of the obtained $P(R)$ distributions exhibit two distinct peaks (see Fig. 9), and hence can only be fitted by the sum of two Gaussian functions, as it is illustrated in the inset of Figure 10. The peak positions of the two individual Gaussians as well as their relative weights in the fitting function are shown in Fig. 10 as a function of the DMSO mole fraction in the surface layer. As is seen, the position of the first peak is composition independent, it is located at about $2.2 \AA$, exactly where the peak of the $P(R)$ distribution is located at the surface of neat water. This peak reflects the fact that neat water-like domains do exist even in the surface layer of water-rich DMSO-water mixtures (as there are circular voids that are surrounded by waters), although their fraction rapidly decreases with increasing DMSO content, as seen from the sharp drop of its weight in the total fitting function (lower panel of 
This document is the Accepted Manuscript version of a Published Work that appeared in final form in J. Phys.: Condens. Matter 28 (2016) 404002 (10pp), copyright @ IOP Science after peer review and technical editing by the publisher. To access the final edited and published work see http://iopscience.iop.org/article/10.1088/0953$\underline{8984 / 28 / 40 / 404002}$

Fig. 10).The second peak, which quickly becomes the dominant feature of the $P(R)$ distribution, and remains the only one in DMSO-rich surfaces, shows a considerable position dependence with increasing DMSO mole fraction. This peak is clearly the counterpart of the one seen when only DMSO molecules are taken into account, and reflects again the same trivial effect of dilution.

The differences seen in the $P(R)$ distributions when only DMSO and when only water molecules are taken into account in the analysis reflects the different dilution mechanisms of the two neat components by the other one [!53] even at the surface of their mixtures. The difference in these mechanisms stems from the fact that although both molecules show higher affinity to unlike than to like neighbours, this effect is stronger for DMSO than for water [!53]. In other words, DMSO likes to be mixed with water more than water likes to be mixed with DMSO. This difference is also seen in the composition dependence of the entropy of mixing of the two components, namely that upon dilution neat DMSO with water the corresponding entropy change is positive, whereas upon diluting neat water with DMSO the entropy of the system decreases [!18,52]. Therefore, in water-rich systems small neat water-like microenvironments can still exist, even at the liquid surface as patches, and therefore the circular vacancies located inside such patches have about the same size than those at the surface of neat water, as seen from the corresponding $P(R)$ distributions. On the other hand, no sign of similar patches formed by DMSO molecules are seen at the surface of DMSO-rich mixtures, as evidenced by the lack of a composition independent peak of the corresponding $P(R)$ distributions.

Finally, it should be noted that the obtained $P(R)$ distributions turned out to be rather similar, and hence they lead to similar conclusions, than those in the bulk liquid phase of these mixtures, apart from one important point. Namely, in the bulk liquid phase the $P(R)$ distributions, obtained when only the water molecules are taken into account, consist of a third peak at composition independent position [!53]. This peak was interpreted as a direct evidence for the existence of strongly hydrogen bonded complexes built up by one DMSO and two water molecules [!53]. The existence of such complexes, usually referred to as DMSO $2 \mathrm{H}_{2} \mathrm{O}$ has been hypothesized in the literature for several decades [!20,35,42,45,46,48]. On the other hand, no such peak is observed in the corresponding $P(R)$ distributions at the liquid surface, indicating that no such complexes exist within the surface layer of DMSO-water mixtures. 
This document is the Accepted Manuscript version of a Published Work that appeared in final form in J. Phys.: Condens. Matter 28 (2016) 404002 (10pp), copyright @ IOP Science after peer review and technical editing by the publisher. To access the final edited and published work see http://iopscience.iop.org/article/10.1088/0953$\underline{8984 / 28 / 40 / 404002}$

\section{Summary and conclusions}

In this paper we have presented a detailed two-dimensional Voronoi analysis of the molecules located at the liquid surface of DMSO-water mixtures of different compositions, aiming at characterising their local lateral environment within the surface layer. Particular attention has been paid to compare the present results with those obtained previously in the bulk liquid phase of these mixtures [!53]. We have found evidences that the molecules prefer to be in a mixed local environment, consisting of both types of molecules, even at the liquid surface, and this preference is even stronger here than in the bulk liquid phase. Unlike in the bulk liquid phase, we have not observed signs of neat-like patches, in which molecules are surrounded by like neighbours, not even in the case of surfaces containing one of the two components in a large excess. On the other hand, we have found voids that are located between solely water molecules at water-rich surfaces. This finding indicates that the different mechanisms corresponding to the dilution of the two neat liquids, already observed in the bulk phase of these mixtures [!53], are valid also at the liquid surface. Finally, we have not found any evidence that strongly hydrogen bonded DMSO $2 \mathrm{H}_{2} \mathrm{O}$ complexes, known to exist in the bulk liquid phase, would be present also at the surface of DMSO-water mixtures.

\section{Acknowledgements}

This work has been supported by the Hungarian OTKA Foundation under Project No. OTKA 104234. 
This document is the Accepted Manuscript version of a Published Work that appeared in final form in J. Phys.: Condens. Matter 28 (2016) 404002 (10pp), copyright @ IOP Science after peer review and technical editing by the publisher. To access the final edited and published work see http://iopscience.iop.org/article/10.1088/0953$\underline{8984 / 28 / 40 / 404002}$

\section{References.}

[1] Martin D and Hauthal G 1975 Dimethyl Sulfoxide (Wiley: New York)

[2] Pfitzner K E and Moffatt J G 1963 J. Am. Chem. Soc. 853027

[3] Lee T V 1991 Comp. Org. Syn. 7291

[4] Tidwell T T 1990 Synthesis 857

[5] Manusco A J, Brownfain D S and Swern D 1979 J. Org. Chem. 444148

[6] Damaskin B B, Tyurin V Y and Dyatkina S L 1991 Elektrokhim 271358

[7] Pegg D E 2007 Methods Mol. Biol. 36839

[8] Foucault A P and Chevolot L 1998 J. Chrom. A 8083

[9] Chakrabarti R and Schutt C E 2001 Gene 271293

[10] Yu Z and Quinn P 1994 Biosci Rep. 14259

[11] Schichman A A and Amey R L 1971 J. Phys. Chem. 7598

[12] del Carmen Grande M, Juliá J A, García M and Marschoff C M 2007 J. Chem. Thermodyn. 391049

[13] Doucet M Y, Calmes-Petrault F and Durand M T 1965 C. R. Acad. Sci. Paris 2601878

[14] Płowaś I Świergiel J and Jadżyn J 2013 J. Chem. Eng. Data 581741

[15] Markarian S A and Terzyan A M 2007 J. Chem. Eng. Data 521704

[16] LeBel R G and Goring D A. 1962 J. Chem. Eng. Data 7100

[17] Cowie J M G and Toporowski P M 1961 Can. J. Chem. 392240

[18] Clever H L and Pigott S P 1971 J. Chem. Thermodyn. 3221

[19] Lam S Y and Benoit R L 1974 Can. J. Chem. 52718

[20] Palaiologou M, Arianas G and Tsierkezos N 2006 . J. Solution Chem. 351551

[21] Egorov G I and Makarov D M 2009 J. Phys. Chem. A. 83693

[22] Sergievskii V V, Skorobogat'ko D S and Rudakov A M 2010 Russ. J. Phys. Chem. A. 84

[23] Rasmussen D H and Mackenzie A P 1968 Nature 2201315

[24] Havemeyer R N 2006 J. Pharm. Sci. 55851

[25] Kaatze U, Pottel R and Schafer M 1989 J. Phys. Chem. 935623

[26] Safford G J, Schaller P C, Leung P S, Doebbler G F, Brady G W and Lyden E F X 1969 J. Chem. Phys. 502140

[27] Brink G and Falk M 1970 J. Mol. Struct. 527 
This document is the Accepted Manuscript version of a Published Work that appeared in final form in J. Phys.: Condens. Matter 28 (2016) 404002 (10pp), copyright @ IOP Science after peer review and technical editing by the publisher. To access the final edited and published work see http://iopscience.iop.org/article/10.1088/0953$\underline{8984 / 28 / 40 / 404002}$

[27] Bertulozza A, Bonora S, Battaglia M A and Monti P 1979 J. Raman Spectr. 8231

[28] Soper A K and Egelstaff P A 1981 Mol. Phys. 42399

[29] Gordalla B C and Zeidler M D 1986 Mol. Phys. 59817

[30] Kaatze U, Brai M, Scholle F D and Pottel R 1990 J. Mol. Liq. 44197

[31] Gordalla B C and Zeidler M D 1991 Mol. Phys. 74975

[32] Soper A K and Luzar A 1992 J. Chem. Phys. 971320

[33] Shin D N, Wijnen J W, Engberts J B F N and Wakisaka A 2001 J. Phys. Chem. B 1056759

[34] Wiewiór P P, Shirota H and Castner E W 2002 J. Chem. Phys. 1164643

[35] Bordallo H N, Herwig K W, Luther B M and Levinger N E 2004 J. Chem. Phys. 12112457

[36] Wulf A and Ludwig R 2006 ChemPhysChem 7266

[37] McLain S E, Soper A K and Luzar A 2007 J. Chem. Phys. 127174515

[38] Noack K, Kiefer J and Leipertz A 2010 ChemPhysChem 11630

[39] Jiang J C, Lin K H, Li S C, Shih P M, Hung K C, Lin S H and Chang H C 2011 J. Chem. Phys. 134044506

[40] Wong D B, Sokolowsky K P, El-Barghouthi M I, Fenn E E, Giammanco C H, Sturlaugson A L and Fayer M D 2012 J. Phys. Chem. B 1165479

[41] Luzar A 1990 J. Mol. Liquids 46221

[42] Vaisman I I and Berkowitz M L 1992 J. Am. Chem. Soc. 1147889

[43] Luzar A and Chandler D 1993 J. Chem. Phys. 988160

[44] Skaf M S 1999 J. Phys. Chem. A 10310719

[45] Borin I A and Skaf M S 1999 J. Chem. Phys. 1106412

[46] Vishnyakov A, Lyubartsev, A P and Laaksonen A 2001 J. Phys. Chem. A 1051702

[47] Skaf M S and Vechi S M 2003 J.Chem. Phys. 1192181

[48] Mancera R L, Chalaris M and Samios J 2004 J. Mol. Liq. 1101473

[49] Harpham M R, Levinger N E and Ladanyi B M 2008 J. Phys. Chem. B 112283

[50] Roy S, Banerjee S, Biyani N, Jana B and Bagchi B 2010 J. Phys. Chem. B 115685

[51] Zhang N, Li W, Chen C and Zuo J 2013 Comput. Theor. Chem. 1017126

[52] Idrissi A, Marekha B, Barj M and Jedlovszky P 2014 J. Phys. Chem. B 1188724

[53] Idrissi A, Marekha B, Kiselev M and Jedlovszky P 2015 Phys. Chem. Chem. Phys. 173470

[54] Kirchner B and Hutte J 2002 Chem. Phys. Lett. 364497

[55] Idrissi A, Polok K, Barj M, Marekha B, Kiselev M and Jedlovszky P 2013 J. Phys. Chem. B 11716157 
This document is the Accepted Manuscript version of a Published Work that appeared in final form in J. Phys.: Condens. Matter 28 (2016) 404002 (10pp), copyright @ IOP Science after peer review and technical editing by the publisher. To access the final edited and published work see http://iopscience.iop.org/article/10.1088/0953$\underline{8984 / 28 / 40 / 404002}$

[56] Karpovich D S and Ray D 1998 J. Phys. Chem. B 102649

[57] Allen H C, Gragson D E and Richmond G L 1999 J. Phys. Chem. B 103660

[58] Tarbuck T L and Richmond G L 2005 J. Phys. Chem. B 10920868

[59] Chen X, Minofar B, Jungwirth P and Allen H C 2010 J. Phys. Chem. B 11415546

[60] Luzar A. 1989 J. Chem. Phys. 913603

[61] Benjamin I 1999 J.Chem. Phys. 1108070

[62] Pojják K, Darvas M, Horvai G and Jedlovszky P 2010 J. Phys. Chem. B 11412207

[63] Pártay L B, Hantal Gy, Jedlovszky P, Vincze Á and Horvai G 2008 J. Comp. Chem. 29945

[64] Hantal G, Darvas M, Pártay L B, Horvai G and Jedlovszky P 2010 J. Phys.: Condens. Matter 22284112

[65] Pártay L B, Horvai G and Jedlovszky P 2010 J. Phys. Chem. C 11421681

[66] Mezei M 2003 J. Mol. Graphics Modell. 21463

[67] Chacón E and Tarazona P 2003 Phys. Rev. Lett. 91166103

[68] Chowdhary J and Ladanyi B M 2006 J. Phys. Chem. B 11015442

[69] Jorge M and Cordeiro M N D S 2007 J. Phys. Chem. C 11117612

[70] Wilard A P and Chandler D 2010 J. Phys. Chem. B 1141954

[71] Jorge M, Jedlovszky P and Cordeiro M N D S 2010 J. Phys. Chem. C. 11411169

[72] Sega M, Kantorovich S S, Jedlovszky P and Jorge M 2013 J. Chem. Phys. 138044110

[73] Medvedev N N 2000 The Voronoi-Delaunay Method in the Structural Investigation of NonCrystalline Systems (SB RAS: Novosibirsk) in Russian

[74] Okabe A, Boots B, Sugihara K and Chiu S N 2000 Spatial Tessellations: Concepts and Applications of Voronoi Diagrams (John Wiley: Chichester)

[75] Voronoi G F 1908 J. Reine Angew. Math. 134198

[76] Jorgensen W L, Chandrashekar J, Madura J D, Impey R and Klein M L 1983 J. Chem. Phys. 79926

[77] Hess B, Bekker H, Berendsen H J C and Fraaije J G E M 1997 J. Comp. Chem. 181463

[78] Allen M P and Tildesley D J 1987 Computer Simulation of Liquids (Clarendon Press: Oxford)

[79] Essman U, Perera L, Berkowitz M L, Darden T, Lee H and Pedersen L G 1995 J. Chem. Phys. 1038577

[80] Hess B, Kutzner C, van der Spoel D and Lindahl E 2008 J. Chem. Theory Comput. 4435

[81] Nosé S 1984 Mol. Phys. 52255

[82] Hoover W G 1985 Phys. Rev. A 311695 
This document is the Accepted Manuscript version of a Published Work that appeared in final form in J. Phys.:

Condens. Matter 28 (2016) 404002 (10pp), copyright @ IOP Science after peer review and technical editing by the publisher. To access the final edited and published work see http://iopscience.iop.org/article/10.1088/0953-

$\underline{8984 / 28 / 40 / 404002}$

[83] Parrinello M and Rahman A 1981 J. Appl. Phys. 527182

[84] Ruocco G, Sampoli M and Vallauri R 1992 J. Chem. Phys. 966167

[85] Jedlovszky P, Medvedev N N and Mezei M 2004 J. Phys. Chem. B 108465

[86] Idrissi A, Damay P, Yukichi K and Jedlovszky P 2008 J. Chem. Phys. 129164512

[87] Idrissi A, Polok K, Gadomski W, Vyalov I, Agapov A, Kiselev M, Barj M and Jedlovszky P 2012 Phys. Chem. Chem. Phys. 145979 
This document is the Accepted Manuscript version of a Published Work that appeared in final form in J. Phys.: Condens. Matter 28 (2016) 404002 (10pp), copyright @ IOP Science after peer review and technical editing by the publisher. To access the final edited and published work see http://iopscience.iop.org/article/10.1088/0953$\underline{8984 / 28 / 40 / 404002}$

\section{Tables}

Table 1. Interaction parameters of the molecular models used.

\begin{tabular}{ccccc}
\hline \hline molecule & interaction site & $\varepsilon / \mathrm{kJ}$ mol-1 & $\sigma / \AA$ & $\mathrm{q} / \mathrm{e}$ \\
\hline \multirow{2}{*}{ DMSO $^{\mathrm{a}}$} & $\mathrm{CH} 3$ & 0.096 & 3.76 & 0.160 \\
& $\mathrm{~S}$ & 1.399 & 3.66 & 0.139 \\
& $\mathrm{O}$ & 0.591 & 2.92 & -0.459 \\
& $\mathrm{H}$ & - & - & 0.52 \\
water $^{\mathrm{b}}$ & $\mathrm{O}$ & 0.6491 & 3.154 & 0.00 \\
& $\mathrm{M}^{\mathrm{c}}$ & - & - & -1.04 \\
\hline \hline
\end{tabular}

${ }^{\mathrm{a}}$ Ref. [!46] ${ }^{\mathrm{b}}$ Ref. [!76] ${ }^{\mathrm{c}}$ Non-atomic interaction site

Table 2. Composition (in terms of DMSO mole fraction) of the surface layer and bulk liquid phase, and average number of water and DMSO molecules per liquid surface in the different systems simulated.

\begin{tabular}{ccccc}
\hline \hline system & $\begin{array}{c}\text { bulk } \\
\text { composition }\end{array}$ & $\begin{array}{c}\text { surface } \\
\text { composition }\end{array}$ & $\begin{array}{c}\text { average number } \\
\text { of water } \\
\text { molecules }\end{array}$ & $\begin{array}{c}\text { average number } \\
\text { of DMSO } \\
\text { molecules }\end{array}$ \\
\hline DMSO000 & 0.000 & 0.00 & 233.3 & 0 \\
DMSO003 & 0.018 & 0.19 & 138.5 & 33.2 \\
DMSO005 & 0.033 & 0.28 & 113.1 & 43.8 \\
DMSO010 & 0.083 & 0.38 & 89.3 & 54.5 \\
DMSO020 & 0.185 & 0.53 & 61.3 & 69.5 \\
DMSO030 & 0.29 & 0.62 & 47.3 & 77.1 \\
DMSO040 & 0.39 & 0.67 & 40.0 & 82.7 \\
DMSO050 & 0.49 & 0.77 & 26.3 & 89.7 \\
DMSO060 & 0.59 & 0.84 & 17.8 & 95.5 \\
DMSO085 & 0.83 & 0.95 & 5.1 & 102.3 \\
DMSO100 & 1.00 & 1.00 & 0 & 105.6 \\
\hline \hline
\end{tabular}


This document is the Accepted Manuscript version of a Published Work that appeared in final form in J. Phys.: Condens. Matter 28 (2016) 404002 (10pp), copyright @ IOP Science after peer review and technical editing by the publisher. To access the final edited and published work see http://iopscience.iop.org/article/10.1088/0953$\underline{8984 / 28 / 40 / 404002}$

\section{Figure captions}

Figure 1. Equilibrium snapshot showing one of the two surfaces of the DMSO020 system present in the basic box, as obtained from the simulation. DMSO and water molecules are shown by green and blue colours, respectively. The molecules constituting the surface layer are marked by larger spheres. The inset shows the $X, Y$ and $Z$ axes of the basic simulation box.

Figure 2. DMSO mole fraction in the surface layer as a function of that in the bulk liquid phase as obtained in the systems simulated (red filled circles). The line connecting the symbols is just a guide to the eye. The solid line shows the composition of the bulk liquid phase, for reference. The inset shows the composition of the surface layer vs. that of the bulk liquid phase as obtained with probe spheres of different size.

Figure 3. Distribution of the area of the VP of the molecules at the surface of DMSO-water mixtures of different compositions. The curves corresponding to the DMSO085, DMSO060, DMSO050, DMSO040, DMSO030, DMSO020, DMSO010, DMSO005, DMSO003, and DMSO000 systems are shifted upwards by $0.025,0.05,0.075,0.1,0.125,0.15,0.175,0.2,0.225$, and 0.25 units, respectively, for clarity.

Figure 4.Fitting the VP area distribution obtained in the DMSO005 system (black filled circles) by one single Gaussian function (blue dashed line) and by the sum of two Gaussian functions (red solid line). The individual Gaussian functions contributing to the fitting function in the latter case are shown by green short dashed lines.

Figure 5. Composition dependence of the peak positions of the individual Gaussian functions used in the two-Gaussian fit of the VP area distributions (top panel), and the relative weights of these individual Gaussians in the fitting function (bottom panel). The lines connecting the symbols are just guides to the eye. 
This document is the Accepted Manuscript version of a Published Work that appeared in final form in J. Phys.: Condens. Matter 28 (2016) 404002 (10pp), copyright @ IOP Science after peer review and technical editing by the publisher. To access the final edited and published work see http://iopscience.iop.org/article/10.1088/0953$\underline{8984 / 28 / 40 / 404002}$

Figure 6. Distribution of the acircularity parameter of the VP of the molecules at the surface of DMSO-water mixtures of different compositions. The curves corresponding to the DMSO085, DMSO060, DMSO050, DMSO040, DMSO030, DMSO020, DMSO010, DMSO005, DMSO003, and DMSO000 systems are shifted upwards by $0.025,0.05,0.075,0.1,0.125,0.15,0.175,0.2$, 0.225 , and 0.25 units, respectively, for clarity. The arrow indicates the slight shift of the distribution peak to smaller values with increasing DMSO concentration.

Figure 7. Distribution of the radius of the circular voids located between the molecules at the surface of DMSO-water mixtures of different compositions. The curves corresponding to the DMSO085, DMSO060, DMSO050, DMSO040, DMSO030, DMSO020, DMSO010, DMSO005, DMSO003, and DMSO000 systems are shifted upwards by 0.025, 0.05, 0.075, 0.1, 0.125, 0.15, $0.175,0.2,0.225$, and 0.25 units, respectively, for clarity. The arrow indicates the shift of the distribution peak to larger values with increasing DMSO concentration. The inset shows the Gaussian fit (red solid line) of the data obtained in the DMSO020 system (open circles).

Figure 8. Distribution of the radius of the circular voids located between the molecules at the surface of DMSO-water mixtures of different compositions, obtained when only DMSO molecules are taken into account in the analysis. The curves corresponding to the DMSO085, DMSO060, DMSO050, DMSO040, DMSO030, DMSO020, DMSO010, DMSO005, and DMSO003 systems are shifted upwards by $0.075,0.15,0.225,0.3,0.375,0.45,0.525,0.6$, and 0.675 units, respectively, for clarity. The arrow indicates the shift of the distribution peak to larger values with decreasing DMSO concentration.

Figure 9. Distribution of the radius of the circular voids located between the molecules at the surface of DMSO-water mixtures of different compositions, obtained when only water molecules are taken into account in the analysis. The curves corresponding to the DMSO060, DMSO050, DMSO040, DMSO030, DMSO020, DMSO010, DMSO005, DMSO003, and DMSO000 systems are shifted upwards by $0.075,0.15,0.225,0.3,0.375,0.45,0.525,0.6$, and 0.675 units, respectively, for clarity. 
This document is the Accepted Manuscript version of a Published Work that appeared in final form in J. Phys.:

Condens. Matter 28 (2016) 404002 (10pp), copyright @ IOP Science after peer review and technical editing by the publisher. To access the final edited and published work see http://iopscience.iop.org/article/10.1088/0953$\underline{8984 / 28 / 40 / 404002}$

Figure 10. Composition dependence of the peak positions of the individual Gaussian functions used in the two-Gaussian fit of the circular void radius distribution, obtained when only water molecules are taken into account in the analysis (top panel), and the relative weights of these individual Gaussians in the fitting function (bottom panel). The lines connecting the symbols are just guides to the eye. The inset shows the fit of the data obtained in the DMSO005 system (filled circles) by the sum of two Gaussian functions (red solid line). The individual Gaussian functions contributing to the fitting function are also shown (green dashed lines). 
This document is the Accepted Manuscript version of a Published Work that appeared in final form in J. Phys.: Condens. Matter 28 (2016) 404002 (10pp), copyright @ IOP Science after peer review and technical editing by the publisher. To access the final edited and published work see http://iopscience.iop.org/article/10.1088/0953-

Figure 1

Fábián et al.

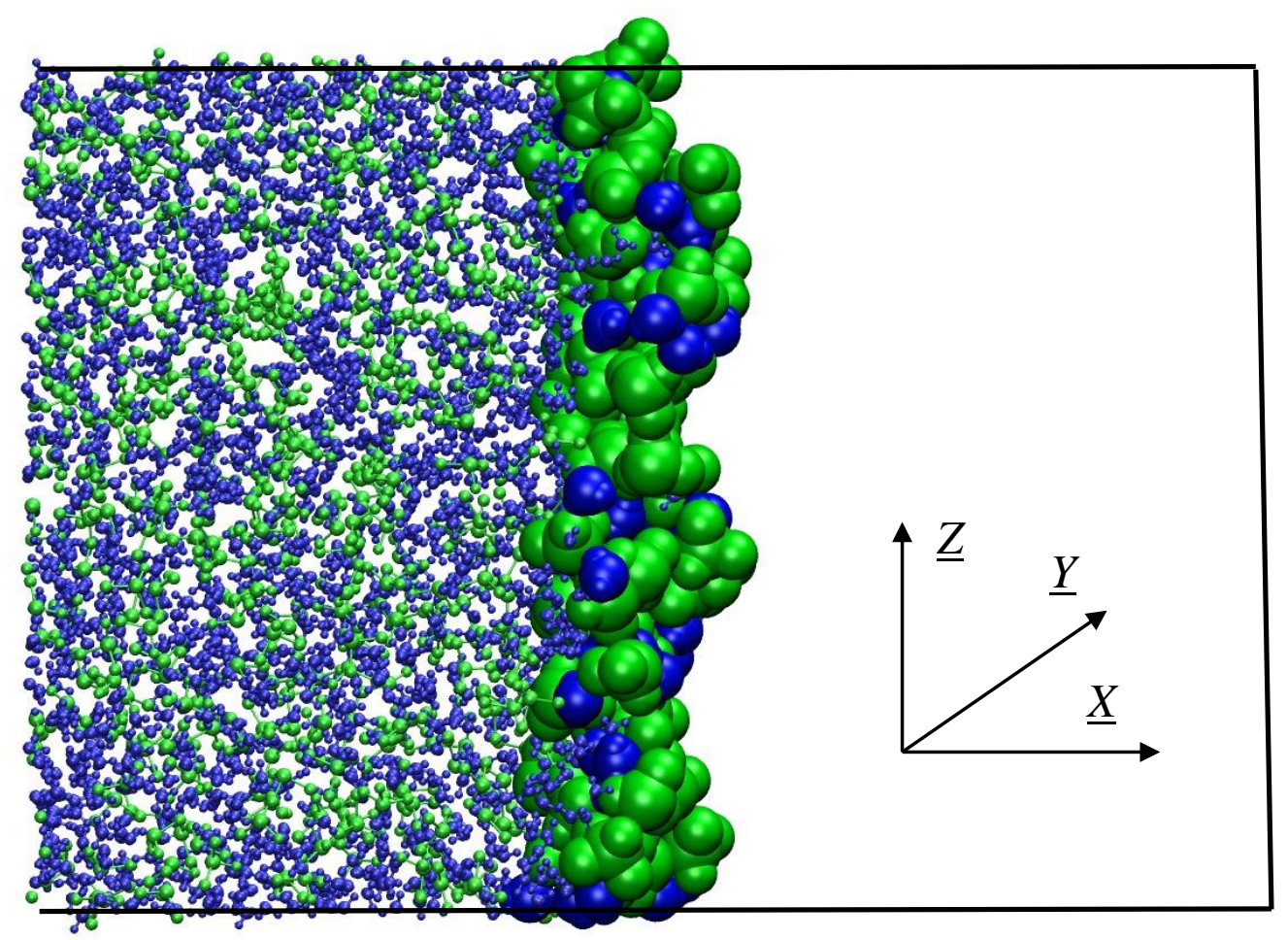


This document is the Accepted Manuscript version of a Published Work that appeared in final form in J. Phys.: Condens. Matter 28 (2016) 404002 (10pp), copyright @ IOP Science after peer review and technical editing by the publisher. To access the final edited and published work see http://iopscience.iop.org/article/10.1088/0953-

Figure 2

Fábián et al.

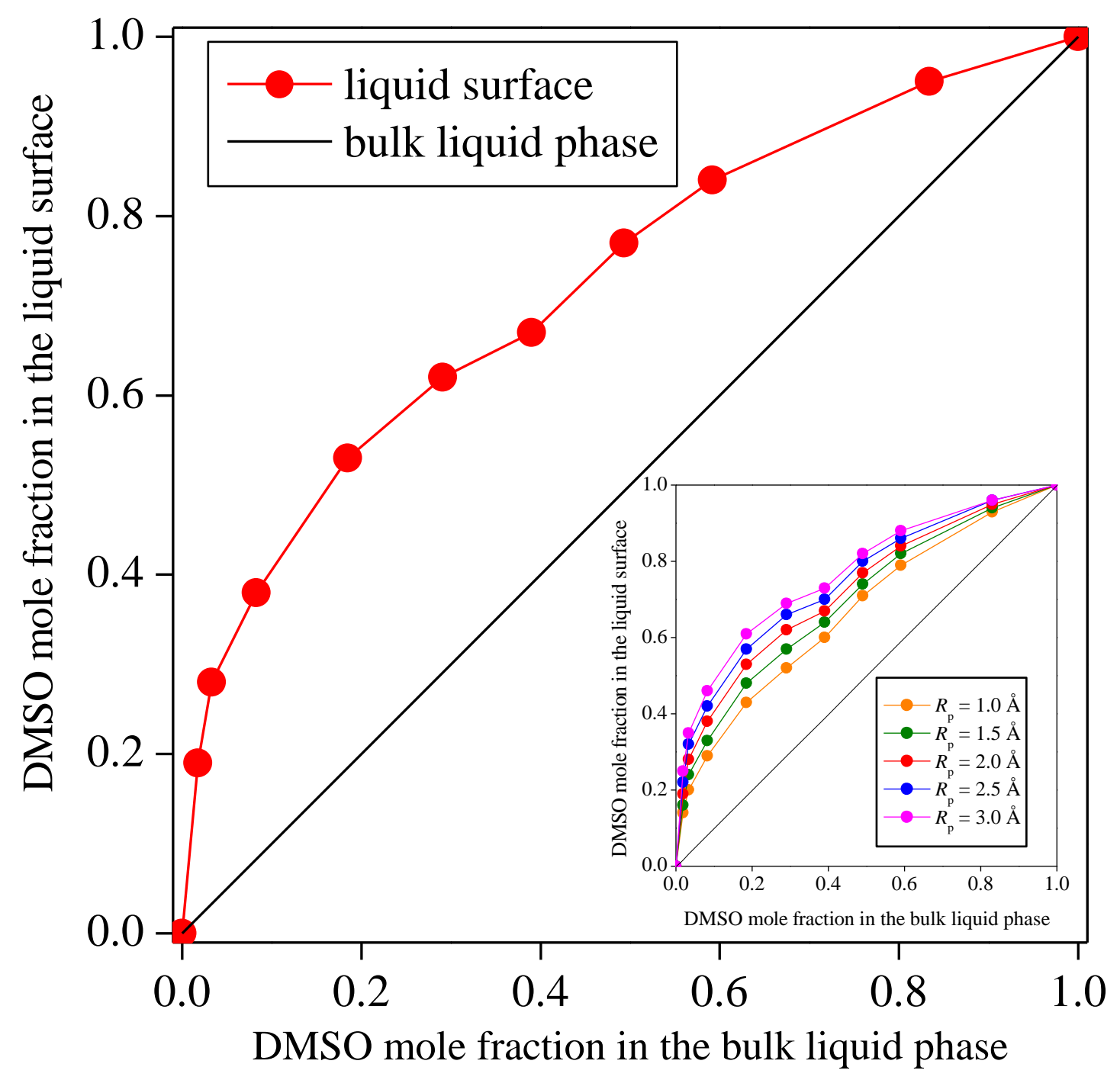


This document is the Accepted Manuscript version of a Published Work that appeared in final form in J. Phys.: Condens. Matter 28 (2016) 404002 (10pp), copyright @ IOP Science after peer review and technical editing by the publisher. To access the final edited and published work see http://iopscience.iop.org/article/10.1088/0953-

Figure 3

Fábián et al.

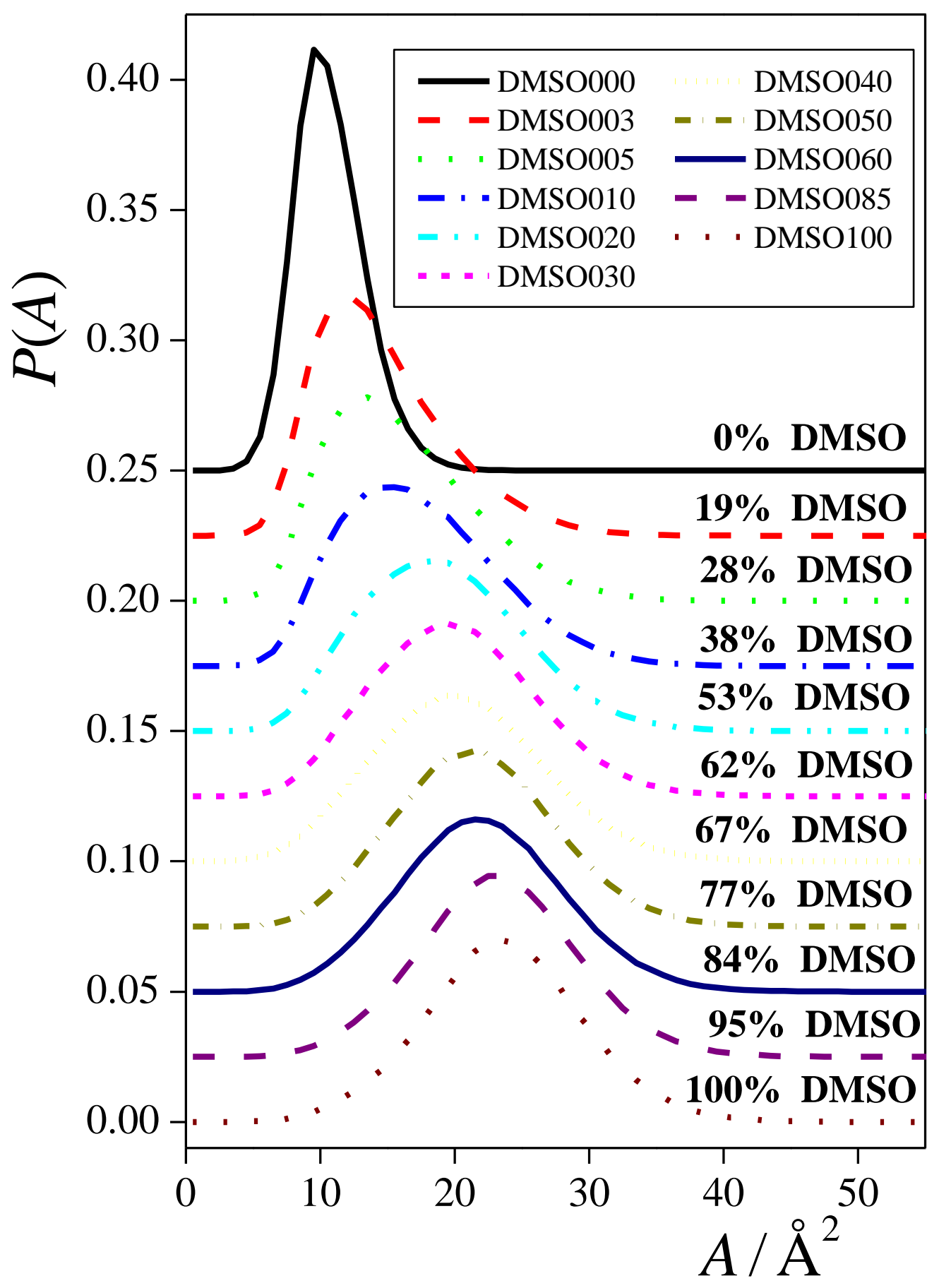


This document is the Accepted Manuscript version of a Published Work that appeared in final form in J. Phys.: Condens. Matter 28 (2016) 404002 (10pp), copyright @ IOP Science after peer review and technical editing by the publisher. To access the final edited and published work see http://iopscience.iop.org/article/10.1088/0953-

Figure 4

Fábián et al.

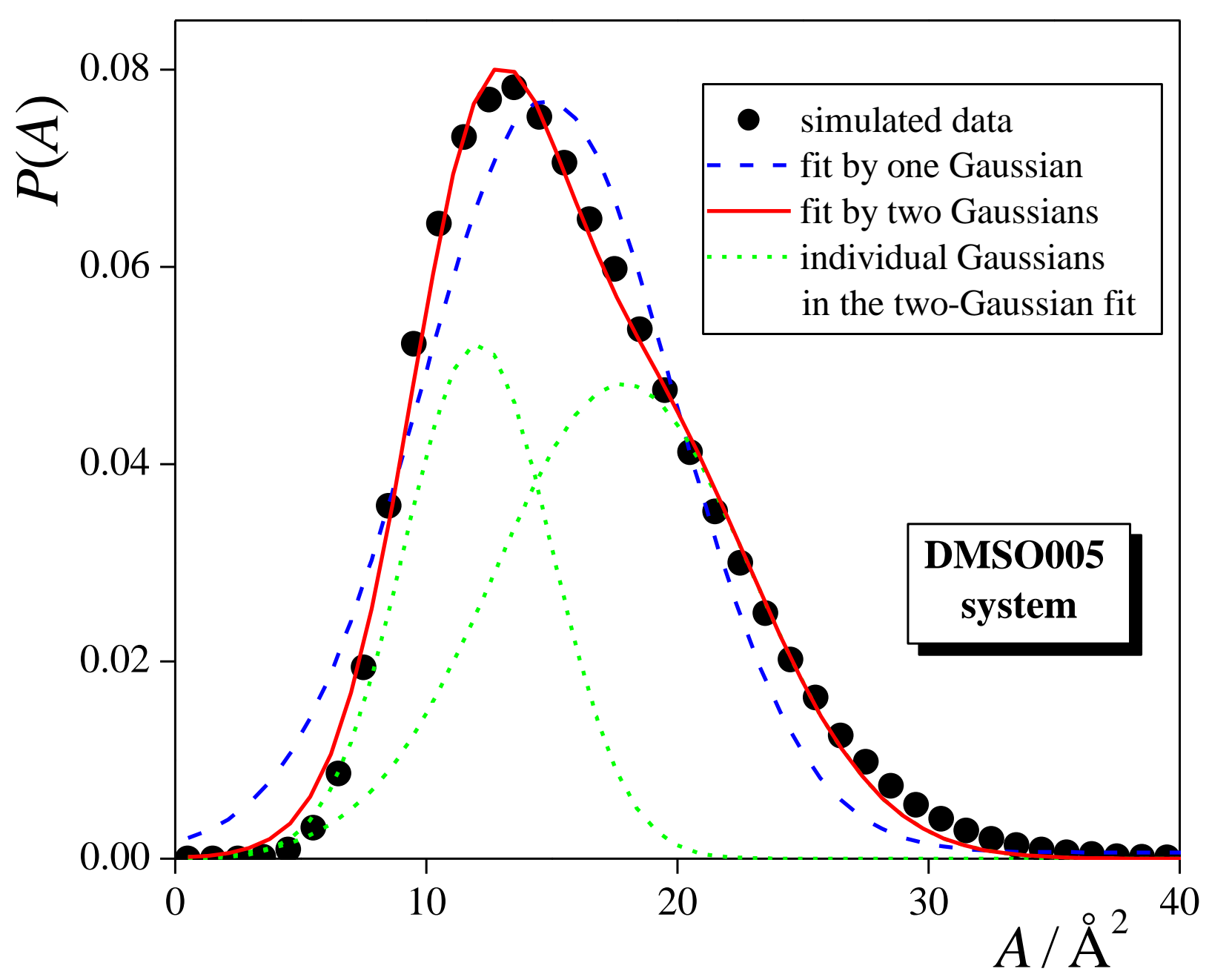


This document is the Accepted Manuscript version of a Published Work that appeared in final form in J. Phys.: Condens. Matter 28 (2016) 404002 (10pp), copyright @ IOP Science after peer review and technical editing by the publisher. To access the final edited and published work see http://iopscience.iop.org/article/10.1088/0953-

Figure 5

Fábián et al.

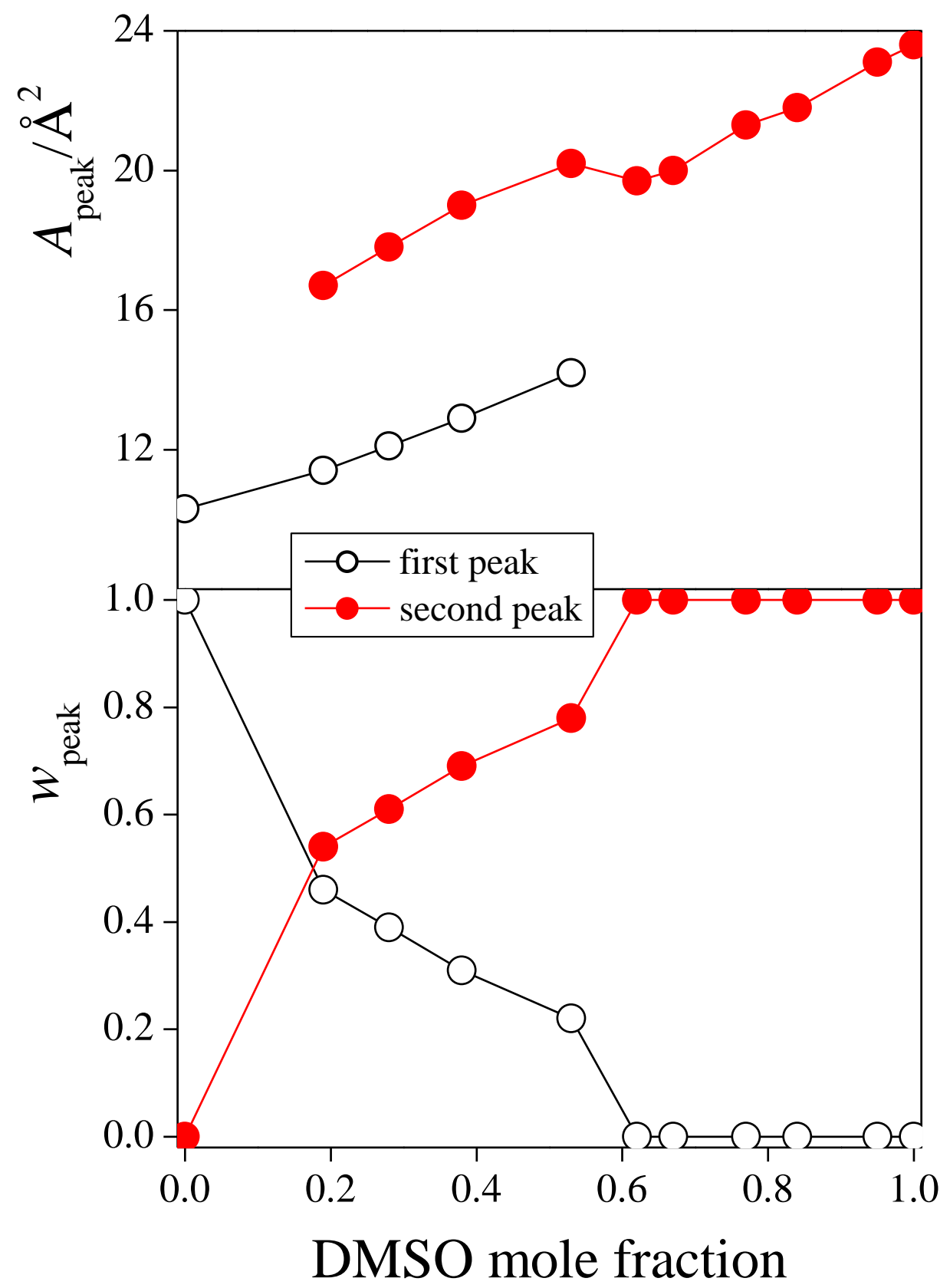


This document is the Accepted Manuscript version of a Published Work that appeared in final form in J. Phys.: Condens. Matter 28 (2016) 404002 (10pp), copyright (C IOP Science after peer review and technical editing by the publisher. To access the final edited and published work see http://iopscience.iop.org/article/10.1088/0953-

Figure 6

Fábián et al.

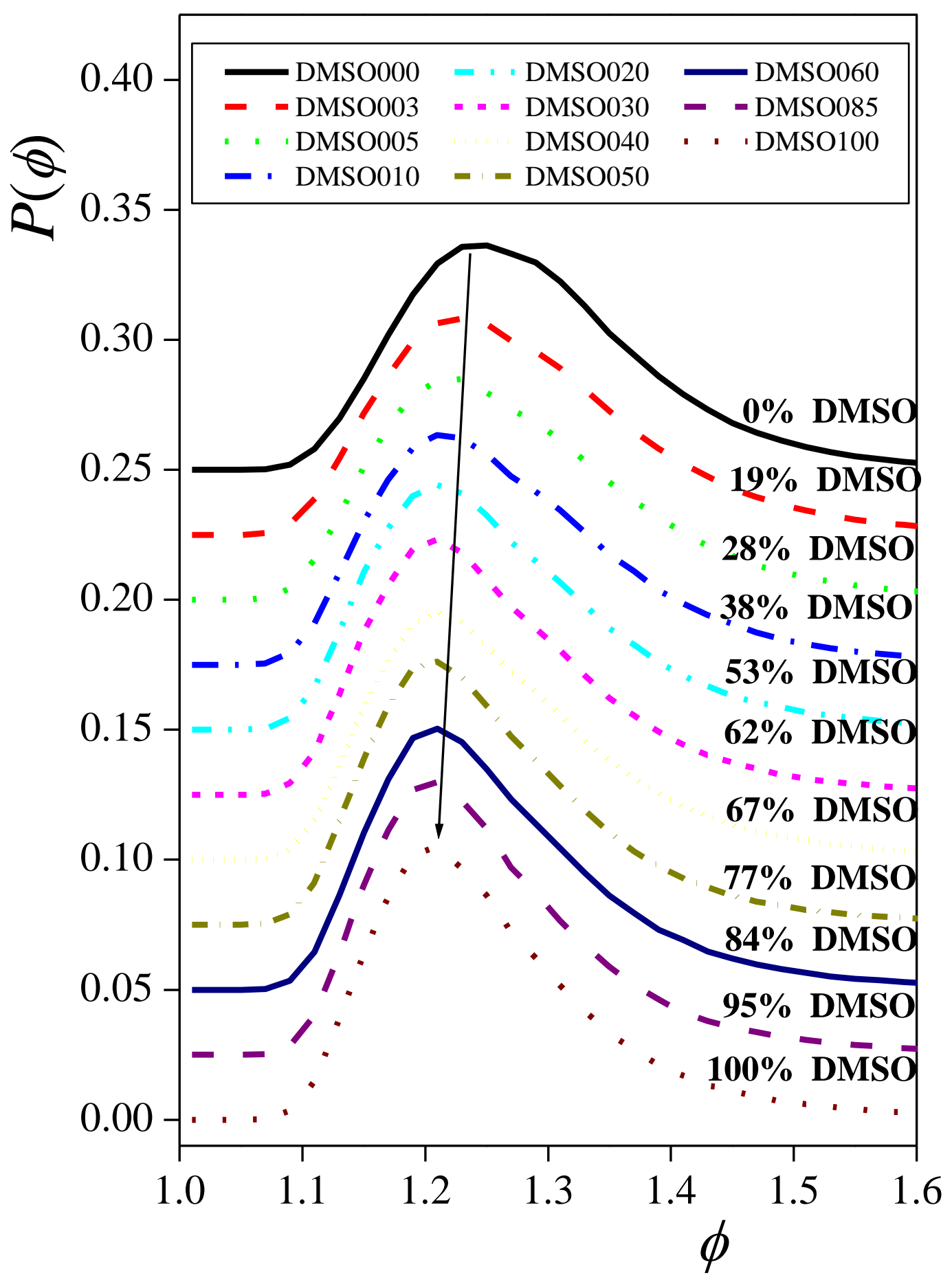


This document is the Accepted Manuscript version of a Published Work that appeared in final form in J. Phys.: Condens. Matter 28 (2016) 404002 (10pp), copyright @ IOP Science after peer review and technical editing by the publisher. To access the final edited and published work see http://iopscience.iop.org/article/10.1088/0953-

Figure 7

Fábián et al.

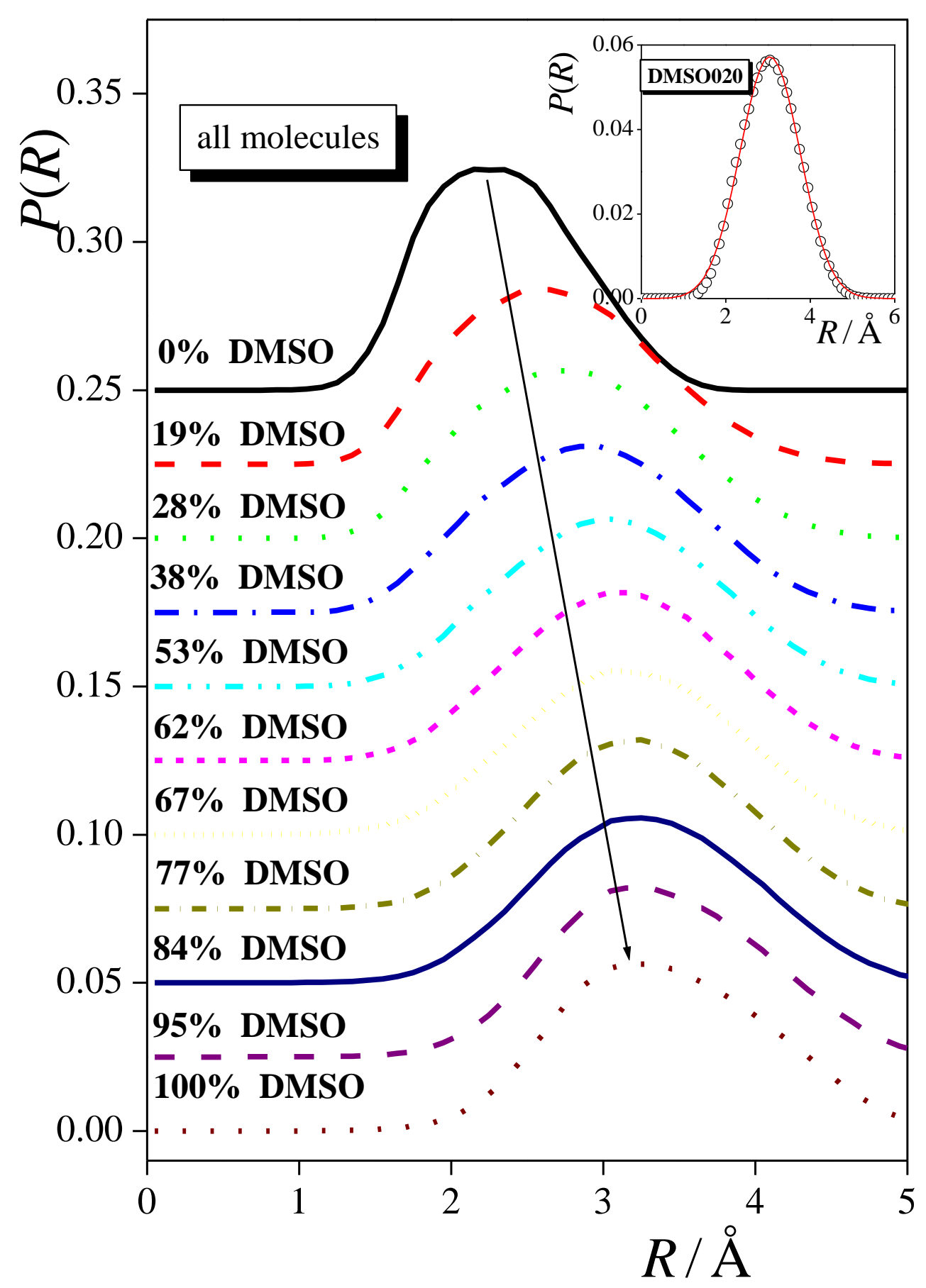


This document is the Accepted Manuscript version of a Published Work that appeared in final form in J. Phys.: Condens. Matter 28 (2016) 404002 (10pp), copyright @ IOP Science after peer review and technical editing by the publisher. To access the final edited and published work see http://iopscience.iop.org/article/10.1088/0953-

Figure 8

Fábián et al.

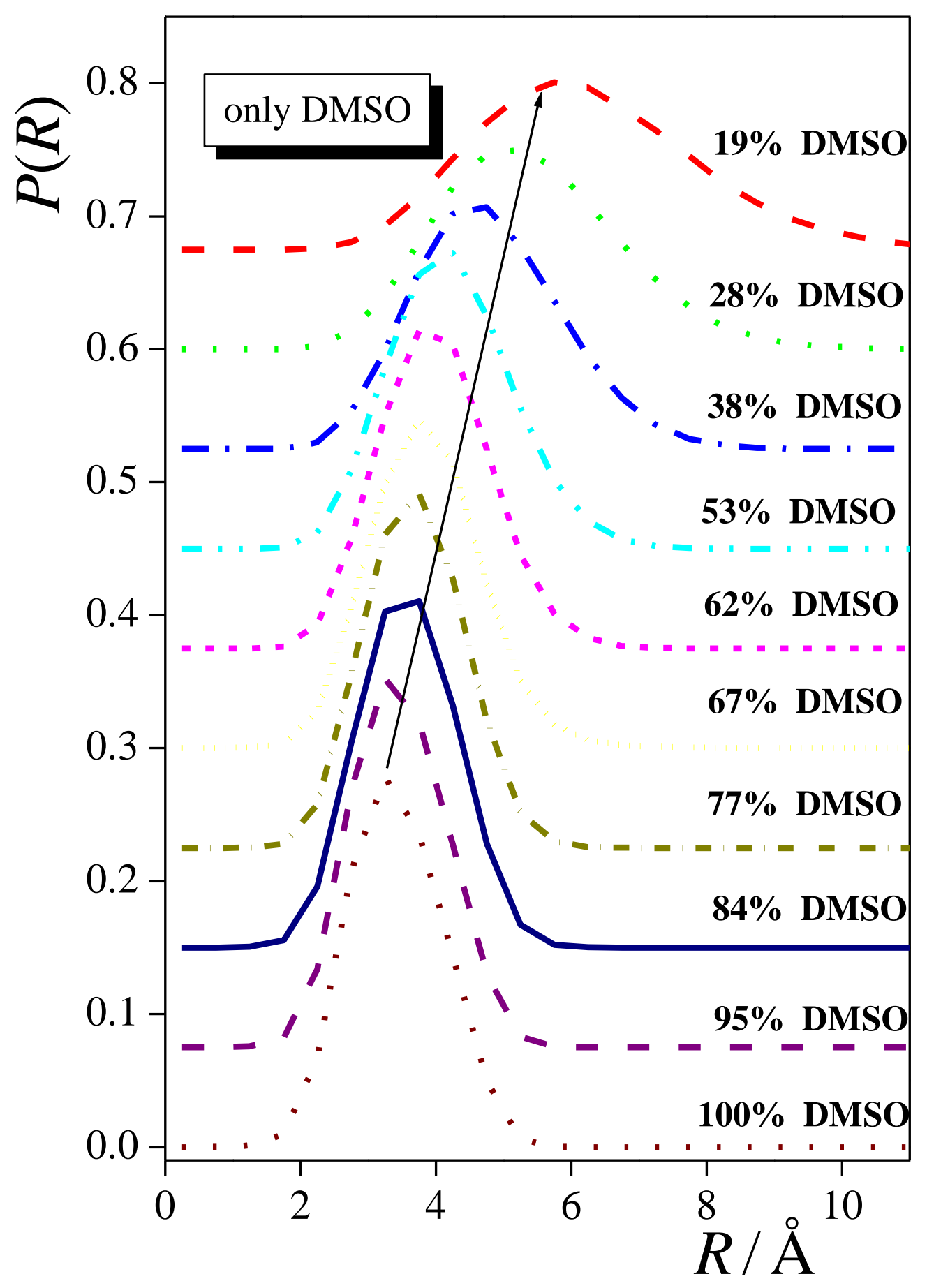


This document is the Accepted Manuscript version of a Published Work that appeared in final form in J. Phys.: Condens. Matter 28 (2016) 404002 (10pp), copyright @ IOP Science after peer review and technical editing by the publisher. To access the final edited and published work see http://iopscience.iop.org/article/10.1088/0953-

Figure 9

Fábián et al.

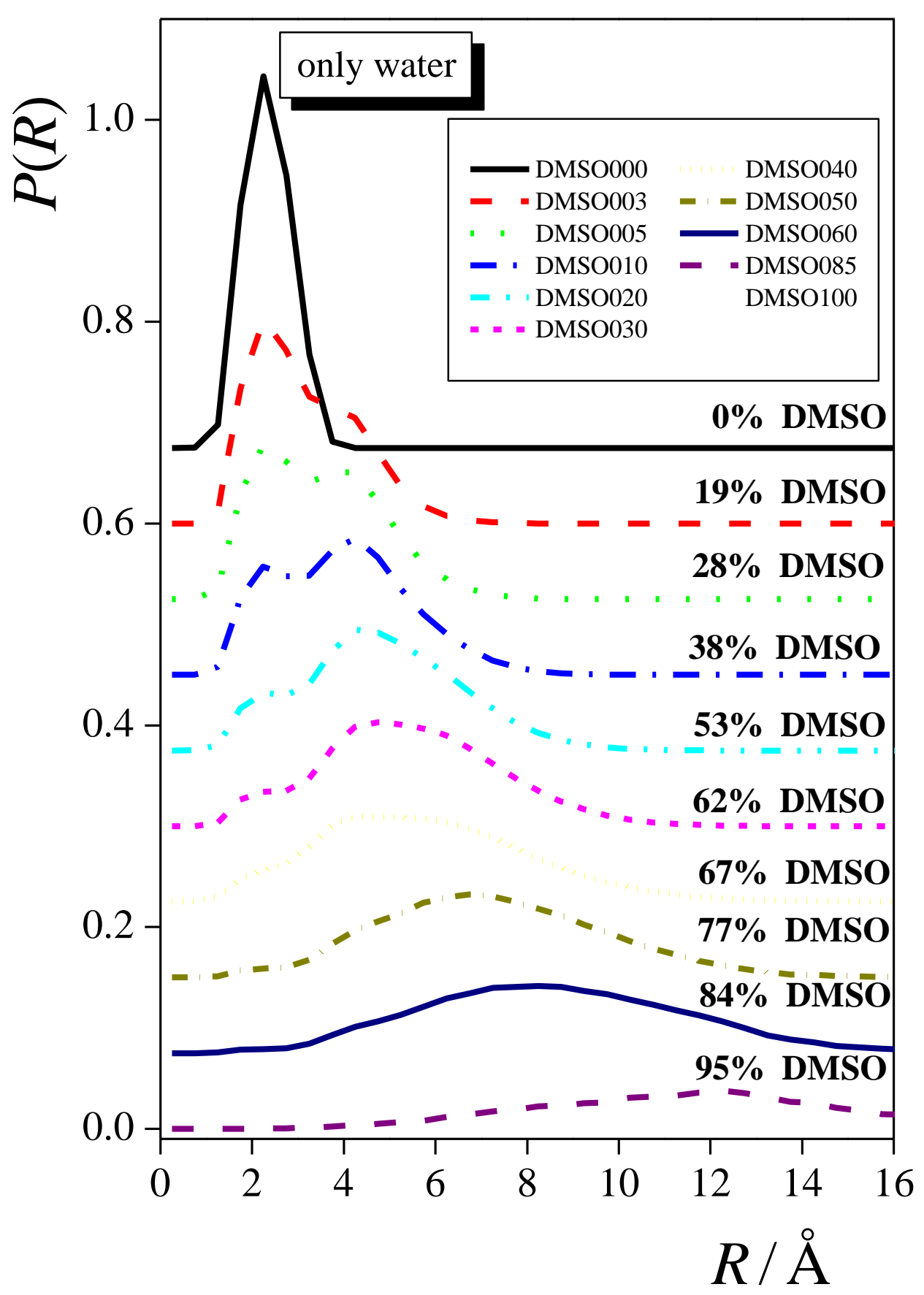


This document is the Accepted Manuscript version of a Published Work that appeared in final form in J. Phys.: Condens. Matter 28 (2016) 404002 (10pp), copyright @ IOP Science after peer review and technical editing by the publisher. To access the final edited and published work see http://iopscience.iop.org/article/10.1088/0953-

Figure 10

Fábián et al.

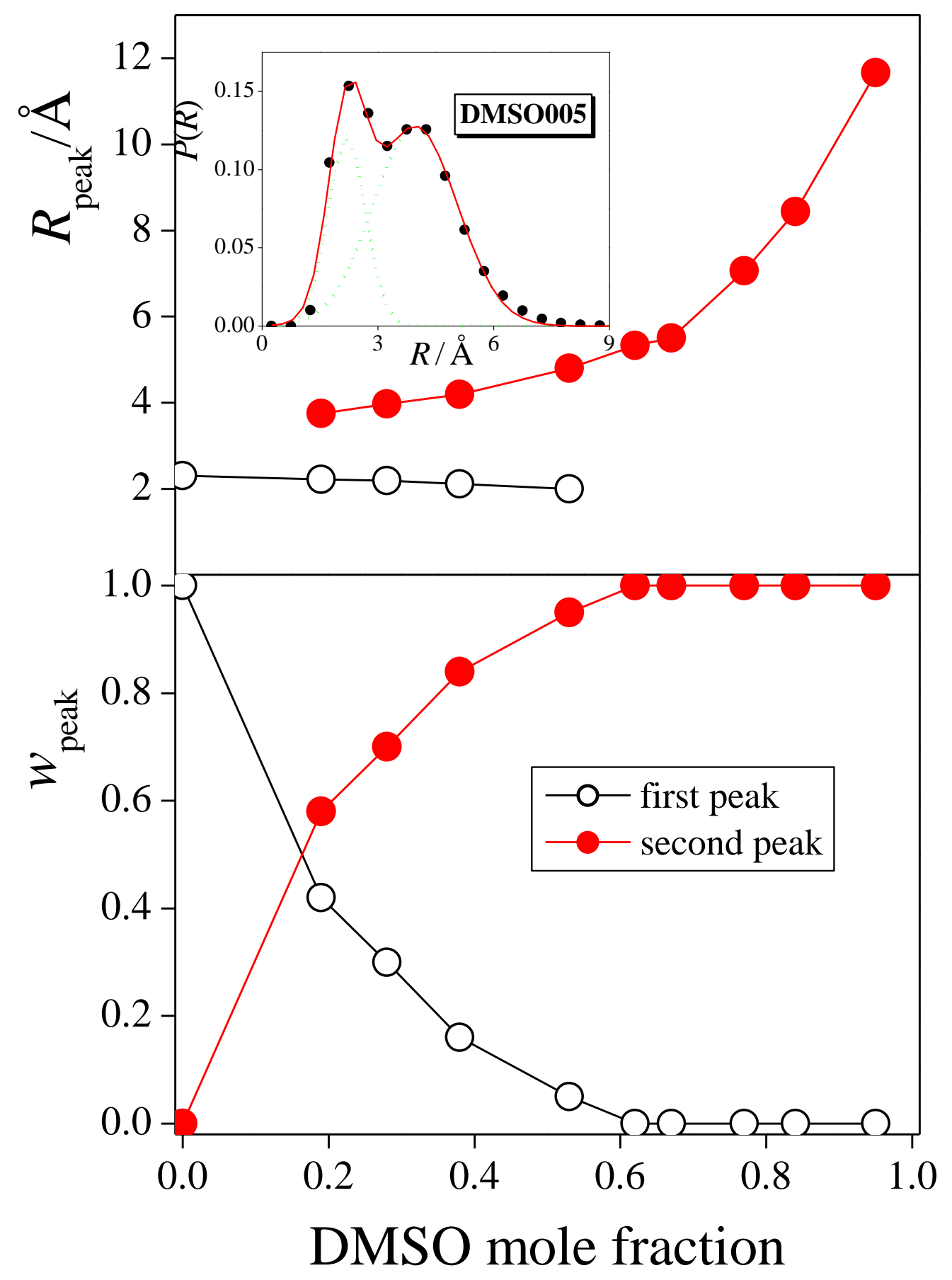


This document is the Accepted Manuscript version of a Published Work that appeared in final form in J. Phys.: Condens. Matter 28 (2016) 404002 (10pp), copyright (C IOP Science after peer review and technical editing by the publisher. To access the final edited and published work see http://iopscience.iop.org/article/10.1088/0953-

Figure $\mathbf{X}$

Fábián et al.

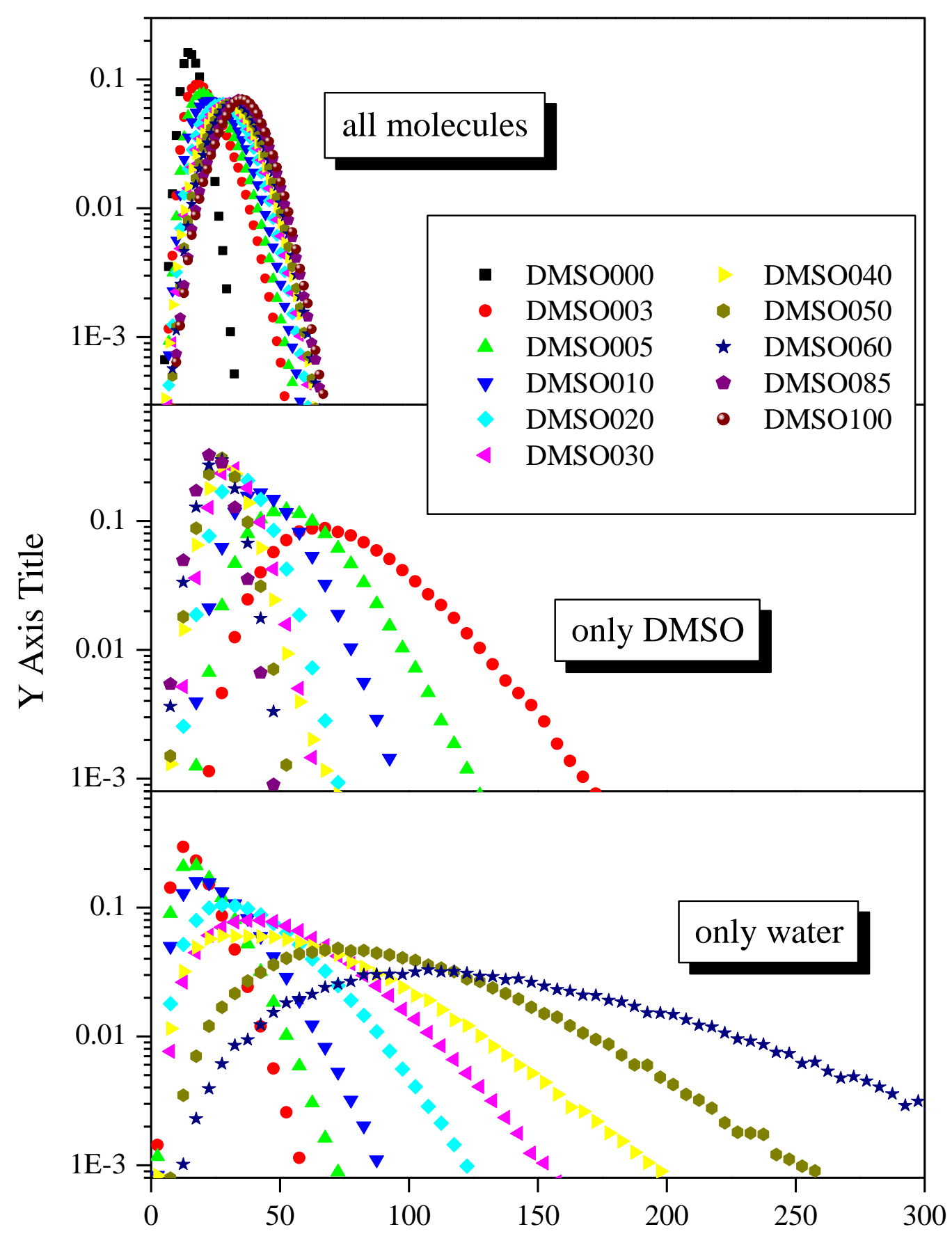

